\title{
Peper An Efficient ANN Interference Cancelation for High Order Modulation over Rayleigh Fading Channel
}

\author{
Fateh Bouguerra and Lamir Saidi \\ LAAAS Laboratory, Department of Electronics, University of Batna 2, Batna, Algeria
}

https://doi.org/10.26636/jtit.2018.125718

\begin{abstract}
High order modulation (HOM) presents a key challenge in increasing spectrum efficiency in $4 \mathrm{G}$ and upcoming 5G communication systems. In this paper, two non-linear adaptive equalizer techniques based on multilayer perceptron (MLP) and radial basis function (RBF) are designed and applied on HOM to optimize its performance despite its high sensitivity to noise and channel distortions. The artificial neural network's (ANN) adaptive equalizer architectures and learning methods are simplified to avoid more complexity and to ensure greater speed in symbol decision making. They will be compared with the following popular adaptive filters: least mean square (LMS) and recursive least squares (RLS), in terms of bit error rate (BER) and minimum square error (MSE) with 16, 64, 128, 256, 512 and 1024 quadrature amplitude modulation (QAM). By that, this work will show the advantage that the MLP equalizer has, in most cases, over RBF and traditional linear equalizers.
\end{abstract}

Keywords-adaptive filter, channel equalization, M-QAM, MLP, $R B F$, symbol decision making.

\section{Introduction}

Many key technologies for enhancing spectrum efficiency are planned for $5 \mathrm{G}$, such as new non-orthogonal access scheme, generalized frequency division access, filter bank multicarrier and universal filtered multicarrier [1], massive multiple-input multiple-output and high order modulation (HOM), e.g. 256 QAM [2]. 256 QAM (8 bits/symbol) increases the maximum peak rate by $33 \%$ compared to 64 QAM (6 bits/symbol). Several studies are conducted that are concerned with employing HOM. In [3], HOM improves the system's capacity in small cells. In [4], transmission performance of HOM-based 256 and 1024 QAM over OFDM is investigated with the impact on error vector magnitude (EVM). A practical FPGA implementation of HOM is already done in [5], using the conventional constant modulus algorithm and LMS equalizers. Even though linear equalizers are widely used, they have poor performance regarding HOM complexity and sensitivity to distortions.

HOM offers better performance than lower order modulation, but still has some external and internal impairments, such as inter-cell interference (ICI) and sensitivity to mul- tipath propagation effects (external), or thermal noise and quality degradation caused by practical manufacturing constraints influencing the EVM (internal). Employing HOM comes with the expense of a higher signal-to-noise (SNR) requirement.

ANNs, being non-linear equalizers, are gaining importance in channel equalization for their flexible architecture, optimization and the learning process [6]. In this paper, multilayer perception (MLP) and radial basis function (RBF) are considered for the simplicity of their architecture and for their ease of use, and especially for their different learning techniques, namely back-propagation, extended Kalman filter, genetic algorithm, and particle swarm optimization [7]. In the case of MLP, we will focus on the back-propagation algorithm. RBF is based on the Euclidian norm. The equalization problem is treated as a classification process that tells to what sets the received symbol belongs, and makes a decision. We will show the effectiveness of the developed MLP and RBF compared to conventional receivers with HOM, by diminishing MSE and BER criteria.

In literature, many types of channels were presented. Different models and distributions were mathematically developed under two categories: channel with line of sight (LOS), and channels with non-line of sight (NLOS). The modeling of NLOS channels is a calculation of physical processes (reflection, diffraction, scattering) affecting the signal during transmission. Nakagami- $m$ represents a generalized distribution that can be applied as a LOS or NLOS channel. Rice and Rayleigh distributions are special cases of Nakagami- $m$ that can be considered as LOS and NLOS channels, respectively [8]. They are mostly used for modeling those channels. In this work, the Rayleigh fading channel is used due to its good and fair approximations of multipath fading in real life. Model generation will be depicted in Section 4.

The equalizers were designed and trained by M-QAM symbols ( $\mathrm{M}$ is modulation order) over an Orthogonal Frequency Division Multiplexing (OFDM) wave carrier in a noisy Rayleigh flat-fading channel. Simulations are carried out using an Intel Core i5 $(2.2 \mathrm{GHz}) 8 \mathrm{~GB}-\mathrm{RAM}$ computer, in the Matlab 2016 environment.

The paper is structured as follows. Section 2 presents the basics of MLP and RBF equalizer designs. Section 3 de- 
scribes the model of the system used for the transmission with HOM over the Rayleigh fading channel. Section 4 illustrates simulation results and presents the discussion. The paper is concluded by Section 5 .

\section{MLP and RBF Equalizers Design}

ANN equalizers are working with either real or complex values. A real ANN has a simpler architecture and its training is fast and not difficult compared to a complex ANN. The simplicity and speed we are striving to achieve are reached by dividing complex modulated symbols into real and imaginary parts, and treating them separately, one after another. Then, the symbols are reshaped at the output in a complex form. The strategy used in the learning process is that total symbols are split randomly between learning $(70 \%)$, validation $(15 \%)$ and test $(15 \%)$, where each step is evaluated with MSE. The architectures are designed to be simple, in order to avoid computational complexity and to minimize the decision time factor. In this case, three layers are fixed: the input layer, the hidden layer and the output layer. The input layer has one input for both real and imaginary values. The hidden layer contains only one layer of neurons (10 for MLP and 20 to 40 for RBF, depending on M-QAM). The output layer has one output only.

\subsection{MLP}

MLP learning is assured by the back-propagation algorithm (simple, fast, and depends on iterative laws). The cost function (MSE) is minimized by adaptation of weight coefficients, through:

$$
j(w)=\frac{1}{2} \sum_{p=1}^{T} \sum_{i=1}^{n}\left|y_{d}(t)-y(t)\right|^{2},
$$

where $y_{d}(t)$ is the desired output, $y(t)$ is the output, $n$ is the number of iterations, and $T$ is the dimension of the training assembly. Weight adaptation (adjustments and updating) is based on the iterative formula:

$$
w_{i j}^{k}(n+1)=w_{i j}^{k}-\mu \frac{\partial j(w)}{\partial w_{i j}^{k}(n)},
$$

where $\mu$ represents the learning step and $k$ is the number of the hidden layer. The activation function is chosen to be hyperbolic-tangent. An initial value of $\mu$ is fixed respecting $\mu<0.1$. Its optimal value is determined gradually through the evolution of the MSE error of MLP.

\section{2. $R B F$}

For RBF, the learning process is divided into two stages: unsupervised nonlinear, where the parameters update techniques are quite fast, and supervised linear problem solving at the output, with the advantage of dodging the local minima issue, often experienced when employing MLP. The training of RBF can be partitioned into three phases.
Firstly, one has to find centers $C_{i}$ of the Gaussian radial functions (activation function). Secondly, widths $\sigma_{i}$ have to be fixed. Finally, the network's weights $\lambda_{j}$ between the radial function and the output layers (Fig. 1) are determined. The simplified function that rules the RBF network, without the independent term reported in formula (21) in [9], is:

$$
F(x)=\sum_{j=1}^{M} \lambda_{j} \mathrm{e}^{-\frac{\left\|x-c_{j}\right\|^{2}}{2 \sigma_{j}^{2}}}+\sum_{i=1}^{d} a_{i} x_{i}
$$

where $\|()$.$\| represents the Euclidean norm, x$ is the input value, $M$ is number of centers $C_{j}, d$ is the length of input variables, $a_{i}$ are the coefficients of the linear terms, and $\mathrm{e}^{(.)}$is the exponential function that represents the nonlinear term.

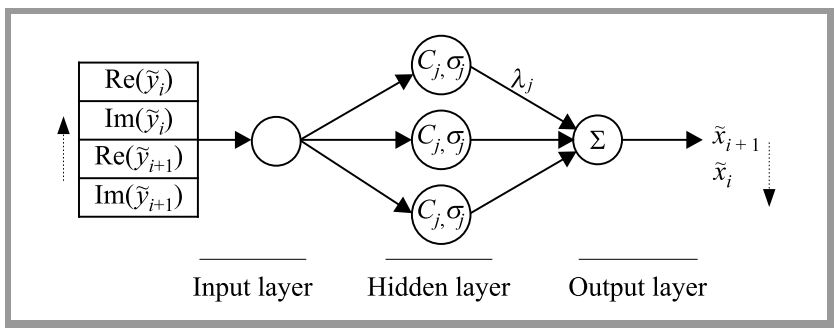

Fig. 1. Simplified RBF structure for symbol decision making with one input, one output and a hidden layer with an adequate number of neurons.

Centers $C_{j}$ are adjusted and fixed utilizing competitive learning (unsupervised) with the Euclidian distance calculations expressed by:

$$
\begin{gathered}
d_{2}\left(x^{i}, C^{j}\right)=\left(x^{i}-C^{j}\right)^{T}\left(x^{i}-C^{j}\right) \\
=\sqrt{\sum_{k=1}^{D}\left(x_{k}^{i}-C_{k}^{j}\right)^{2}}, \\
C^{k}(t+1)=C^{k}(t)+\alpha(t)\left(x^{i}-C^{k}\right),
\end{gathered}
$$

where $\alpha(t)$ is the time adaptation factor, with $0<\alpha(t)<1$, and $\sigma_{j}$ is calculated with:

$$
\sigma=\frac{d_{\max }}{\sqrt{2 M}}
$$

where $d_{\max }$ is the maximum distance between any couple of centroids.

When $C_{j}$ and $\sigma_{j}$ are fixed, Eq. (3) becomes linear, and $\lambda_{j}$ is estimated by the pseudoinverse.

Table 1 summarizes the architecture design and all parameters of MLP and RBF equalizers for all modulation schemes.

For better performance and fair comparison, filter length of LMS has $10 w_{n}$ coefficients, and its step $\mu$ is fixed for each 
M-QAM separately (Table 2). Similarly, RLS filter length has $10 w_{n}$ coefficients, and $\lambda_{R L S}=0.9$ for all M-QAM.

Table 1

Summary of ANN equalizers parameters

\begin{tabular}{|c|c|c|}
\hline Parameters & MLP & $\mathrm{RBF}$ \\
\hline Criterion function & MSE & MSE \\
\hline Hidden layers & 1 & 1 \\
\hline $\begin{array}{l}\text { Hidden layer } \\
\text { neurons }\end{array}$ & 10 & $20 \ldots 40$ \\
\hline $\begin{array}{l}\text { Input layer } \\
\text { neurons }\end{array}$ & 1 & 1 \\
\hline $\begin{array}{l}\text { Output layer } \\
\text { neurons }\end{array}$ & 1 & 1 \\
\hline Parameters & $\begin{array}{c}\text { Weights } w \\
\text { Step } \mu \\
\text { Iteration } n \\
\end{array}$ & $\begin{array}{c}\text { Centroids } C_{i} \\
\text { Widths } \sigma_{i} \\
\text { Weights } \lambda_{i} \\
\end{array}$ \\
\hline Algorithm & Back-propagation & Euclidian norm \\
\hline $\begin{array}{l}\text { Activation } \\
\text { function }\end{array}$ & Hyperbolic-tangent & Gaussian \\
\hline Learning & Supervised & $\begin{array}{c}\text { Supervised } \\
+ \text { unsupervised }\end{array}$ \\
\hline
\end{tabular}

Table 2

LMS parameters

\begin{tabular}{|c|c|c|}
\hline M-QAM & Step $\mu$ & $w_{n}$ number \\
\hline \hline 16 & 0.0039 & 10 \\
64 & 0.0031 & 10 \\
128 & 0.0021 & 10 \\
256 & $4.54 \cdot 10^{-4}$ & 10 \\
512 & $3 \cdot 10^{-4}$ & 10 \\
1024 & $9.99 \cdot 10^{-5}$ & 10 \\
\hline
\end{tabular}

\section{System Model}

In the transmitter part, 300,000 sequences of a random signal $x(n)$ are generated with zero-mean and unit variance, and are supposed to be independent and identically distributed [10]. $x(n)$ is modulated with HOM and shaped with OFDM carriers. In M-QAM, we will have 300000/K symbols, where $K=\log _{2}(M)$ is the number of bits per symbol. $M=16,64,128,256,512,1024$. After modulation, the received signal can be expressed by:

$$
\tilde{y}(n)=x(n) \cdot H+\eta(n),
$$

where $x(n)$ is the signal emitted through $H$ ( $H$ is the Rayleigh flat-fading channel transfer function), and $\eta(n)$ is a complex additive white Gaussian noise generated with the energy per bit-to-noise power spectral density ratio $E_{b} / N_{0}=1: 28 \mathrm{~dB}$, depending on $M$.

The Rayleigh flat-fading channel $H$ is considered as a modeled finite impulse-response filter that can be obtained from a random, uncorrelated, complex Gaussian processes.

In the receiver part, $\tilde{y}(n)$ in Eq. (7) is reconstructed by removing the cyclic prefix, applying the fast Fourier transform and converting parallel stream data to a serial form. After OFDM demodulation, comes the delicate part of equalizing with the use of LMS, RLS, MLP and RBF at different times, and calculating MSE of each of the above for different M-QAM and different SNRs. Finally, the symbols are demodulated to bits to calculate BER. Figure 2 represents the model for symbol decision making for all equalizers referred to in this paper.

\section{Simulations and Results}

All HOM schemes and their MSE values are depicted in Figs. 3 and 4, where MSE values are expressed in terms of multiple SNRs. LMS and RLS have excessive MSE values in the order of $10^{2}$ to $10^{4}$. This shows that linear equalizers have poor performance, particularly on low SNR values.

MLP and RBF show great resistance to channel effects, where MSE is in the order of $10^{-4}$ to 5 with 16,64 and 128 QAM, and in the order of 5 to $10^{2}$ for 256,512 and 1024 QAM. In 16 and 512 QAM, MSE of MLP and RBF are almost the same. In 64 and 128 QAM, MSE of $\mathrm{RBF}$ is better, and in 256 and 1024 QAM, MSE of MLP is better.

In terms of BER, we first lay out the theoretical BER of every M-QAM. Here, the theoretical BER is considered as a reference to measure the effectiveness of equalizers coupled with signal degrading factors, such as SNR, channel

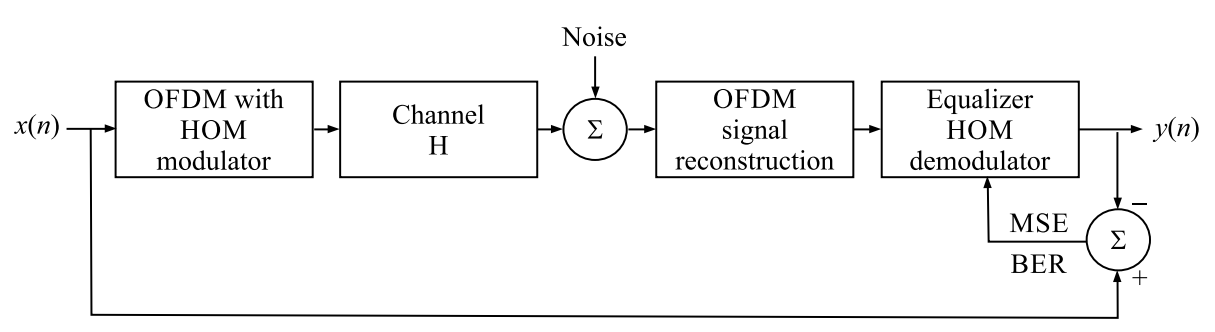

Fig. 2. Channel equalization model. 

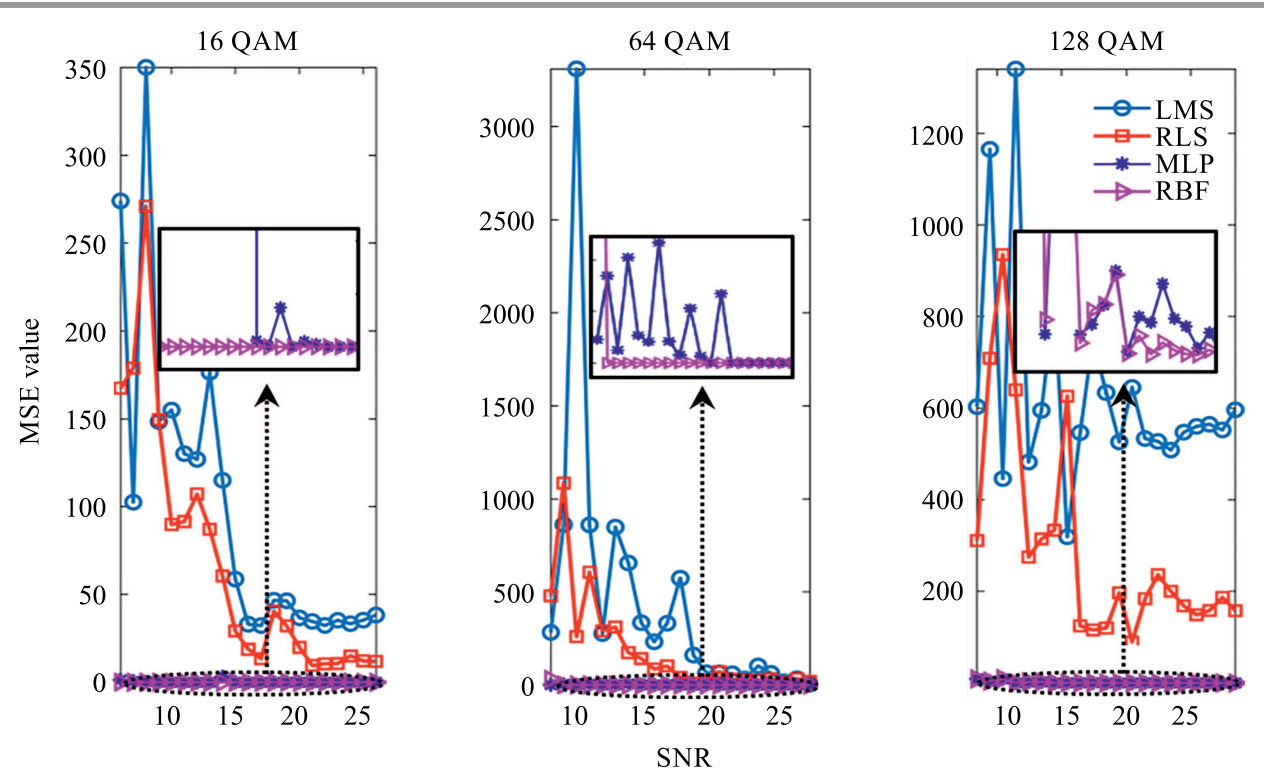

Fig. 3. Effect of SNR on MSE of equalizers, with 16, 64, and 128 QAM. (For color pictures visit www.nit.eu/publications/ journal-jtit)
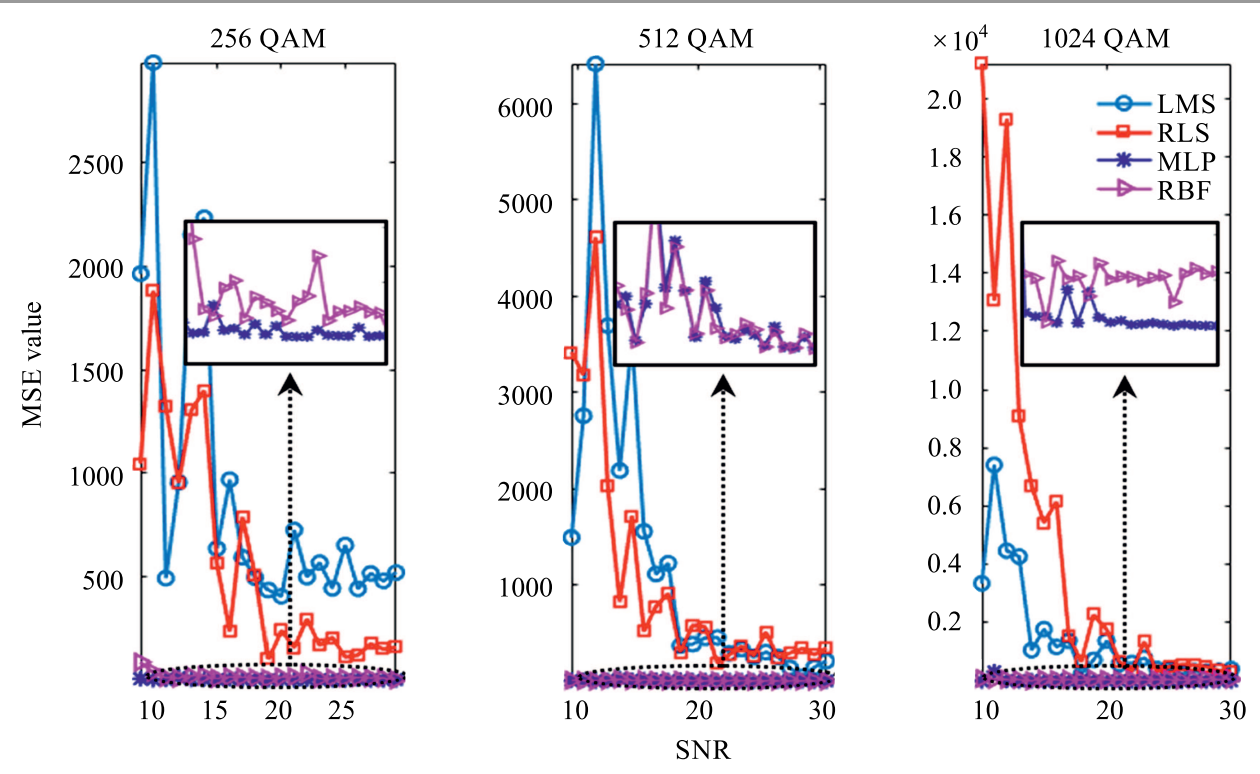

Fig. 4. Effect of SNR on MSE of equalizers, with 256, 512, and 1024 QAM.

type, and sensitivity of the modulation scheme used. The theoretical BER is calculated via equation [11]:

$$
\begin{gathered}
P_{b}=\frac{2}{\sqrt{M} \log _{2} \sqrt{M}} \sum_{K=1}^{\log _{2} \sqrt{M}} \\
\sum_{i=0}^{\left(1-2^{-K}\right) \sqrt{M}-1}\left\{(-1)^{\left.\left\lfloor\frac{i 2^{K-1}}{\sqrt{M}}\right\rfloor\left(2^{K-1}-\left\lfloor\frac{i 2^{K-1}}{\sqrt{M}}+\frac{1}{2}\right\rfloor\right) Q(\phi)\right\}}\right. \\
\phi=\left((2 i+1) \sqrt{\frac{6 \log _{2} M}{2(M-1)} \frac{E_{b}}{N_{0}}}\right)
\end{gathered}
$$

where $P_{b}$ is the BER and $Q(x)$ is the $Q$ function given by:

$$
\begin{aligned}
Q(x)=\frac{1}{\sqrt{2 \pi}} \int_{x}^{\infty} \mathrm{e}^{\frac{-t^{2}}{2}} \mathrm{~d} t & \\
& =\frac{1}{\sqrt{\pi}} \int_{x}^{\infty} \mathrm{e}^{-z^{2}} \mathrm{~d} z=\frac{\operatorname{erfc}(x)}{2},
\end{aligned}
$$

where $\operatorname{erfc}(x)$ is the complementary error function, which is accessible in Matlab software.

With 16 QAM (Fig. 5), RLS and LMS are starting to diverge from the theoretical curve at $11 \mathrm{~dB}$, at the same $E_{b} / N_{0}$ value a slight improvement is noticed in the case of MLP and RBF. At $E_{b} / N_{0}=12 \mathrm{~dB}$, an improvement 
of $1 \mathrm{~dB}$ is observed for both. The two have identical performance in this modulation scheme.

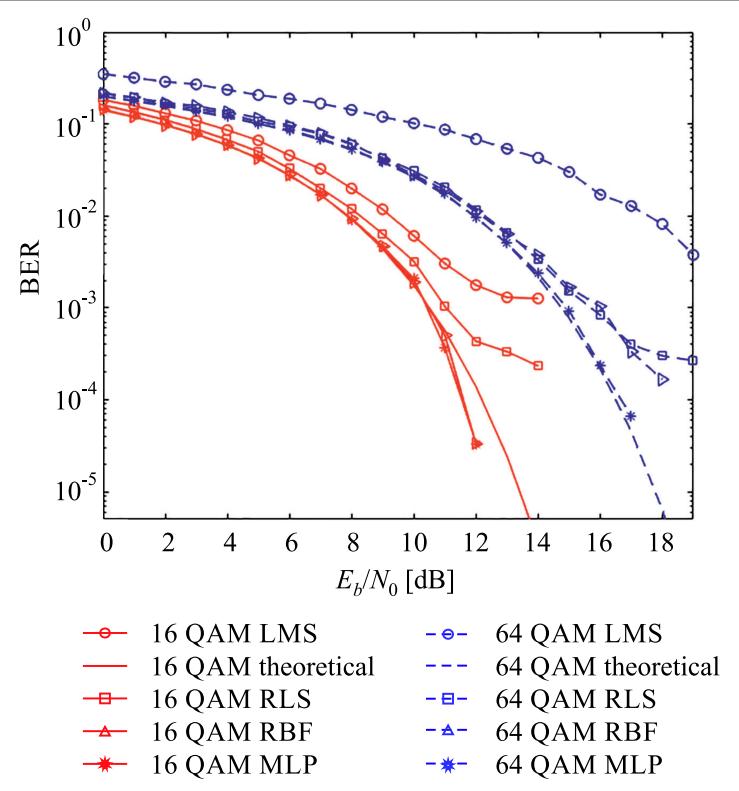

Fig. 5. Equalizer BER performance curves with 16 and 64 QAM.

With 64 QAM (Fig. 5), LMS performance is not good enough for the theoretical curve, RLS and RBF are better but start to diverge at $15 \mathrm{~dB}$. MLP is performing as the theoretical curve, and is the most suited for 64 QAM.

With 128 QAM (Fig. 6), LMS and RLS start to diverge from $13 \mathrm{~dB}$ and $18 \mathrm{~dB}$, respectively. MLP is performing better than linear equalizers, gaining almost $1 \mathrm{~dB}$, but RBF is the most suited for this scheme of modulation by improving by almost $2 \mathrm{~dB}$ compared to the theoretical curve. With 256 QAM (Fig. 6), MLP and RBF are performing

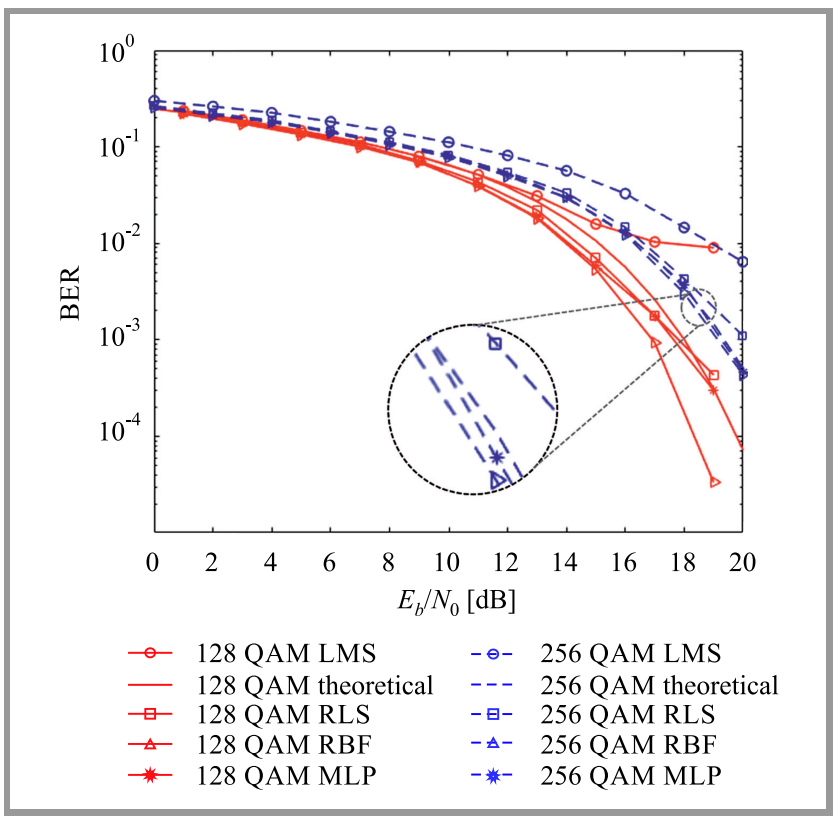

Fig. 6. Equalizer BER performance curves with 128 and 256 QAM. nearly the same as the theoretical curve. A slight improvement is noticed on RBF performance compared to MLP. RLS is better than LMS but starts to diverge at $18 \mathrm{~dB}$.

With 512 and 1024 QAM, spectral efficiency is really high ( 9 and 10 bits per symbol, respectively). As mentioned before, they are sensitive to excessive noise and need more SNR to be investigated compared to lower case modulations. Their performance is shown in Fig. 7. At 512 QAM, the two linear equalizers are diverging, $\mathrm{RBF}$ is trailing the theoretical curve but diverges at $23 \mathrm{~dB}$. MLP is performing really good to gain $1.5 \mathrm{~dB}$, and is better suited to $512 \mathrm{QAM}$ than others.

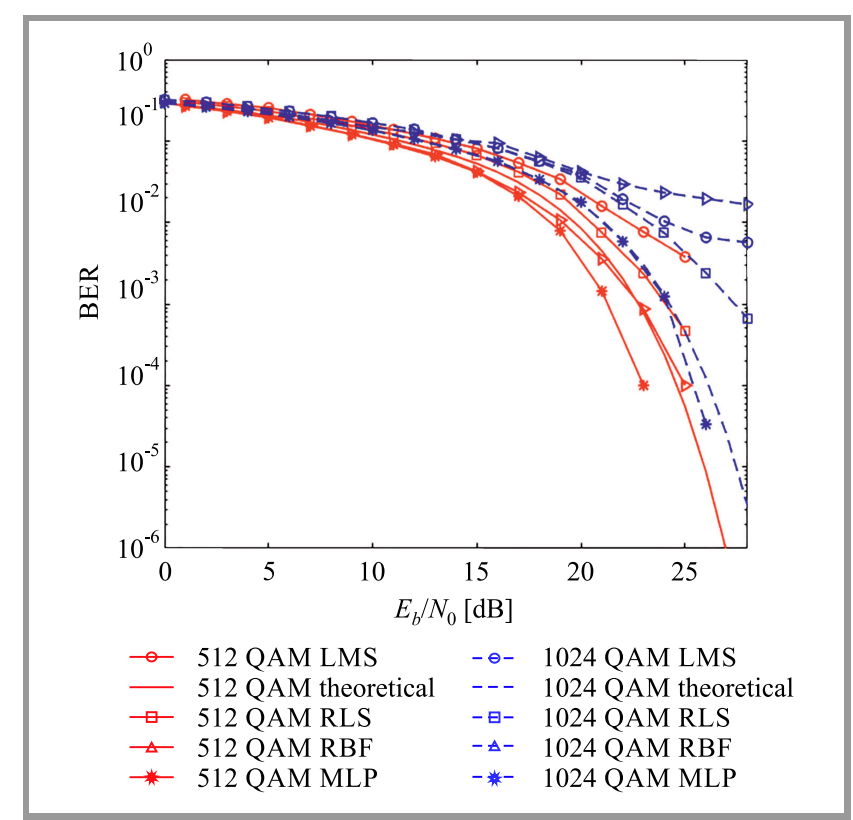

Fig. 7. Equalizer BER performance curves with 512 and 1024 QAM.

With 1024 QAM, MLP is more efficient than RLS, LMS and RBF (Fig. 7) which are not trailing the theoretical curve. RBF performance is poorer than that of LMS and RLS. We tried to train it differently and expand the architecture of RBF by adding more neurons (up to 40 neurons). Unfortunately, there was no improvement. MLP is most suited for this high spectral efficiency.

The developed neural equalizers show great resistance and excellent robustness in terms of adaptation. Symbols are appropriately equalized from the starting point with a better BER, in most cases, than theoretical simulation. When the channel is in a diminutive SNR state, MLP and RBF equalizers do not suffer from any performance degradation, and they are more appropriate for this type of channel coupled with HOM. However, RLS and LMS equalizers offer better performance for low and moderate levels of noise with HOM.

Table 3 represents the profiling of equalizer algorithms during the processing of data (all sequences and all stages of training). Initialization and MSE calculations are not considered. The average of 10 measurements is reported in the table for each algorithm. 256 QAM is chosen be- 
cause - in terms of BER - the equalizers are performing almost similarly. LMS has the shortest duration because of its simplicity. RLS is more complex and it requires more time, which means that it performs similarly to MLP. The latter, at a much better speed, can have a good result for both MSE and BER. RBF takes a long time, since its architecture design is extended (more neurons) to meet better BER results.

Table 3

Profiling the equalizer speed performance with $256 \mathrm{QAM}$ at $E_{b} / N_{0}=20 \mathrm{~dB}$

\begin{tabular}{|c|c|c|c|c|}
\hline Equalizers & LMS & RLS & MLP & RBF \\
\hline \hline Time $[\mathrm{s}]$ & 0.239644 & 0.451853 & 0.542335 & 0.972604 \\
\hline$w_{n} /$ neurons & 10 & 10 & 10 & 20 \\
\hline
\end{tabular}

\section{Conclusion}

In this paper, MLP and RBF equalizers are developed in the simplest form for HOM equalization, serving as a key for achieving more spectral efficiency. It is shown through simulation results that MSE values of the two ANN equalizers are negligible in comparison with LMS and RLS. Also the improved BER, in most M-QAM scenarios, offers great performance in HOM processing, despite its complexity (compared to linear equalizers). These models are more reliable and efficient in terms of canceling noise (MSE criterion) and error rate performance (BER criterion).

MLP is better suited than RBF with 64, 512 and 1024 QAM, and is performing almost identically to RBF with 16 and 256 QAM. RBF is performing well only with 128 QAM. Through the planning of small cells (cancelation of ICI), HOM associated with MLP equalizer are an accessible option for enhancing spectral efficiency and for increasing data rate peaks. Further investigations are to be performed to augment the simplification of learning strategies, architectures and complexity levels.

\section{References}

[1] Y. Tao, L. Liu, S. Liu, and Z. Zhang, "A survey: Several technologies of non-orthogonal transmission for 5G", China Commun., vol. 12, no. 10, pp. 1-15, 2015 (doi: 10.1109/CC.2015.7315054).

[2] Y. Kishiyama, A. Benjebbour, T. Nakamura, and H. Ishii, "Future steps of LTE-A: evolution toward integration of local area and wide area systems", IEEE Wirel. Commun., vol. 20, no. 1, pp. 12-18, 2013 (doi: 10.1109/MWC.2013.6472194).

[3] A. Darwish and A. S. Ibrahim, "Capacity improvement via indoor small cells", in Proc. Int. Wirel. Commun. and Mob. Comput. Conf. IWCMC 2014 , Nicosia, Cyprus, 2014, pp. 69-73 (doi: 10.1109/IWCMC.2014.6906334).

[4] M. Iwamoto, S. Matsuoka, H. Iwasaki, and H. Otsuka, "Transmission performance of OFDM with 1024-QAM in the presence of EVM degradation", in Proc. 2014 IEEE Asia Pacific Conf. on Wirel. and Mob., Bali, Indonesia, 2014, pp. 12-16 (doi: 10.1109/APWiMob.2014.6920262).

[5] S. Ma and Y. Chen, "FPGA implementation of high-throughput complex adaptive equalizer for QAM receiver", in Proc. 8th Int. Conf. on Wirel. Commun., Netw. and Mob. Comput., Shanghai, China, 2012, pp. 1-4 (doi: 10.1109/WiCOM.2012.6478527).
[6] K. Burse, R. N. Yadav, and S. C. Shrivastava, "Channel equalization using neural networks: a review", IEEE Trans. on Syst., Man, and Cybernet., Part C (Appl. and Rev.), vol. 40, no. 3, pp. 352-357, 2010 (doi: 10.1109/TSMCC.2009.2038279).

[7] A. Rubaai and P. Young, "Hardware/software implementation of fuzzy-neural-network self-learning control methods for brushless DC motor drives", IEEE Trans. on Industry Appl., vol. 52, no. 1, pp. 414-424, 2016 (doi: 10.1109/TIA.2015.2468191).

[8] A. Goldsmith, Wireless Communications. Cambridge University Press, 2005 (ISBN: 9780511841224 , doi: 10.1017/CBO9780511841224).

[9] F. Bouguerra, I. Benacer, and L. Saidi, "MLP and RBF symbol tracking with 16 QAM modulation over multipath distorted channel", in Proc. Int. Conf. on Adv. Syst. and Elec. Technol. IC_ASET 2017, Hammamet, Tunisia, 2017, pp. 182-187 (doi: 10.1109/ASET.2017.7983688).

[10] H. Cai, Q. Zhang, Q. Li, and J. Qin, "Proactive monitoring via jamming for rate maximization over MIMO Rayleigh fading channels", IEEE Commun. Lett., vol. 21, no. 9, pp. 2021-2024, 2017 (doi: 10.1109/LCOMM.2017.2715337).

[11] K. Cho and D. Yoon, "On the general BER expression of one- and two-dimensional amplitude modulations", IEEE Trans. on Commun., vol. 50, no. 7, pp. 1074-1080, 2002 (doi: 10.1109/TCOMM.2002.800818).

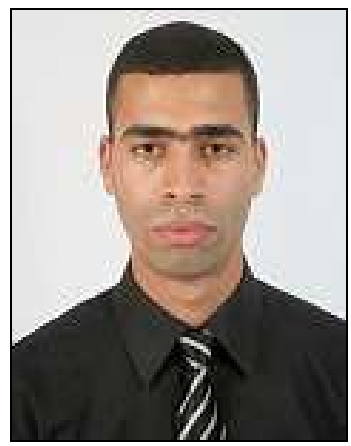

Fateh Bouguerra received his B.Eng. in Electronics and Communication Engineering in 2006 and M.Sc. (Eng.) in Microwave and Telecommunication in 2011 from University of Batna 2. Now he is pursuing his Ph.D. degree as a researcher in the field of Mobile Communications. His interests include digital signal processing, wireless communication networks, artificial intelligence, and biomedical engineering.

E-mail: Bouguerra_fateh@yahoo.fr

LAAAS Laboratory

Department of Electronics

University of Batna 2

Batna, Algeria

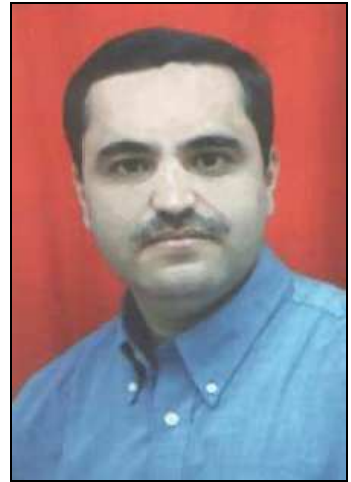

Lamir Saidi received his Ph.D. from University of Savoie, France, in 1996. Currently, he is a full professor at the Electronics Engineering Department, University of Batna 2, Algeria. $\mathrm{He}$ is at the head of the Advanced Automatic and Systems Analysis Laboratory. His interests include digital signal processing and communication systems.

E-mail: 1.saidi@univ-batna2.dz

LAAAS Laboratory

Department of Electronics

University of Batna 2

Batna, Algeria 